eCommons@AKU

June 2015

\title{
Role of evidence-based public health in controlling emerging infectious diseases
}

Tawfik A. M. Khoja

Waris Qidwai

Aga Khan University, waris.qidwai@aku.edu

Mohamed S. Hussein

Lesley Pocock

Follow this and additional works at: https://ecommons.aku.edu/pakistan_fhs_mc_fam_med Part of the Family Medicine Commons

\section{Recommended Citation}

Khoja, T., Qidwai, W., Hussein, M. S., Pocock, L. (2015). Role of evidence-based public health in controlling emerging infectious diseases. Middle East Journal of Family Medicine, 13(4), 28-32.

Available at: https://ecommons.aku.edu/pakistan_fhs_mc_fam_med/90 


\title{
Role of Evidence-Based Public Health in Controlling Emerging Infectious Diseases
}

\author{
Tawfik A. M. Khoja (1) \\ Waris Qidwai (2) \\ Mohamed S. Hussein (3) \\ Lesley Pocock (4)
}

(1) Prof. Tawfik A. M. Khoja,

MBBS, DPHC, FRCGP, FFPH, FRCP (UK)

Director General Executive Board,

Health Ministers Council for Cooperation Council

Professor of Public Health

Family \& Community Physician Consultant

(2) Dr. Waris Qidwai

MBBS MCPS FCPS MRCGP (Int) FCGP (Sri Lanka

Professor \& Chairman

Family Medicine Department

Aga Khan University, Karachi

(3) Dr. Mohamed S. Hussein,

Dr.PH, M.PH, M.SC.,

Riyadh, Saudi Arabia

(4) Lesley Pocock

Publisher and Managing Director

medi+WORLD International

Australia

\section{Correspondence:}

Waris Qidwai,

Professor and Chairman, Family Medicine Department

Aga Khan University Karachi Pakistan

Tel: (92-21) 3486-4843, 3486-4814

Fax: (92-21) 3493-4294, 3493-2095

Email: waris.qidwai@aku.edu

\section{Introduction}

Emerging infectious diseases are on the rise and pose a growing challenge to Health Care Providers around the world. They include infectious diseases that have not occurred in humans before, diseases that have occurred previously but affected only small numbers of people in isolated places such as AIDS and Ebola hemorrhagic fever(1) or diseases that have occurred throughout human history but have only recently been recognized as distinct diseases due to an infectious agent, such as Lyme disease and gastric ulcers.

Today we live in the era of evidence based medicine (EBM) that entails an approach to health care, which promotes the collection, interpretation, and integration of valid, important and applicable patient-reported, clinicianobserved, and research-derived evidence. The best available evidence, moderated by patient circumstances and preferences, is applied to improve the quality of clinical judgments, to ensure Patient safety and facilitate costeffective health care. (2) Evidence-Based public health is defined as the development, implementation and evaluation of effective programs and policies in public health through application of principles of scientific reasoning, including systematic uses of data and information systems, and appropriate use of behavioral science theory and program planning models. $(3,4,5)$ (Table 1) 
Table 1: Key Differences between Evidence-Based Medicine and Evidence-Based Public Health (ED)

\begin{tabular}{|c|c|c|}
\hline Characteristic & $\begin{array}{c}\text { Evidence-Based } \\
\text { Medicine }\end{array}$ & $\begin{array}{c}\text { Evidence-Based } \\
\text { Public Health }\end{array}$ \\
\hline Quality of evidence & Experimental studies & $\begin{array}{c}\text { Observational and quasi- } \\
\text { experimental studies }\end{array}$ \\
\hline $\begin{array}{c}\text { Volume of evidence } \\
\text { intervention to } \\
\text { outcome }\end{array}$ & Larger & Smaller \\
\hline $\begin{array}{c}\text { Professional training } \\
\text { certification and/or } \\
\text { licensing }\end{array}$ & $\begin{array}{c}\text { Less formal, no standard } \\
\text { certification }\end{array}$ \\
\hline Decision making & Individual & Team \\
\hline
\end{tabular}

It is important to apply principles of Evidence-Based Public Health(6) to meet the challenge of emerging infectious diseases. This approach has several advantages which include incorporating the best existing medical interventions with clinical practice whenever suitable evidence about its usefulness is available. It provides guidance to best use resources through identifying interventions that work and defers those lacking evidence for their effectiveness. It helps in finding a common language and general rules for defining the efficacy of available intervention. It provides grounds for improving the graduate studies and continuous medical education programs.(7)

Benefits of implementing Evidence-Based emerging disease practices include improving Health Professionals knowledge, their understanding of research and its methods, confidence in managing clinical situations, computer literacy and data searching skills. It allows group problem solving and teaching and it offers more effective use of resources. It allows better communication with the patient about the rationale behind treatment and juniors can contribute as well as seniors to improve team work.(7)

Barriers to evidence based practice in Emerging Diseases include difficulty in providing best evidence based service that may not be possible within limited resources.

No credible health professional could deny that sound evidence should be an integral part of clinical decision making. The demand for up to date information to inform care and treatment highlights the crucial role of research and development in the modern health service. However, within an ordinary health system, Health Practitioners have not always been able to underpin their actions with robust research findings. (8)
The potential barriers for use of Evidence-Based decision making in emerging diseases include lack of leadership in setting a clear and focused agenda and lack of a long term view (horizon) for program implementation and evaluation. External pressure drives the process away from an Evidence-Based approach and inadequate training in key public health disciplines is an issue. Lack of time to gather information, analyze data and renew the literature for evidence are some of the other challenges. There is also lack of comprehensive, up-to-date information on the effectiveness of programs and policies and lack of data on the effectiveness of certain public health interactions or for special populations.

Evidence-Based Public Health acts on good practice guidelines, incorporating expertjudgments with appropriate, systematic research. There exists a need in public health to identify the scientific basis for efficacy of policies and programs, change evidence into recommendations, and increase evidence used in public health practice. (7)

Policy-makers should invest in scientifically proven and cost-effective remedies with regards to emerging diseases. Scientific basis for efficiency of interventions in programs, practices, or policies, can be used as justification for selection of a certain course of action and allocation of funding and other resources. Patient safety should be addressed through evidence based public health approaches. (9) 
Tools to support Evidence-Based Public

Health

\section{Health Impact Assessment (HIA)}

HIA is an approach to assess burden on health and the potential of health improvements by modifying underlying conditions.(10)

It is a combination of procedures, methods, and tools by which a policy, program, or project may be judged as to its potential effects on the health of a population and the distribution of those effects within the population.

Itrequiresahigh degree ofinterdisciplinaryandinter-sectoral collaboration, well-trained Practitioners, appropriate data sets for analysis, and funders that recognize the value of the collaborations and the information.

Because of their focus on specific policy options facing decision-makers, it is more likely than other evidencebased decision tools to affect decisions outside the health sector.

It educates decision-makers about how the public's health is strongly influenced by many decisions in spheres outside health.

\section{Systematic Reviews}

A systematic review(11) is a formal process that identifies all of the relevant scientific studies on a topic, assesses their quality, individually and collectively, and sums up their results.

Systematic approaches for summarizing scientific evidence and linking that evidence to practice and policy recommendations increase the transparency, understandability, and credibility of recommendations.

Systematic reviews make it easier for practitioners and policymakers to understand all of the relevant information that is available, how it was collected and assembled, and how the conclusions and recommendations relate to the information that was reviewed.

In the health sphere, reviews now summarize information on the efficacy of medical treatments, clinical preventive services, public health interventions and policies, and related social policies.

\section{Portfolio of Tools}

A range of techniques and tools are evolving in this area. Some of the important ones include the following:

A) Participatory research: performing research in collaboration with those affected by the issue under study for the purpose of taking action or making change has the potential to increase the relevance of research findings and their subsequent use in communities. (Figure 1)
B) Increasing collection and reporting of qualitative information about the context in which research studies were conducted will provide users with more information about whether particular strategies are likely to be feasible and useful in local contexts as well as providing a basis for allowing systematic reviews to assess the impact of context on results.

C) Economic evaluations of public health interventions will provide critical information about costs and value.

D) Finally, decision analytic and other modeling approaches can be very helpful in systematically collecting and analyzing available data, comparing the value of two or more decision options and assessing the importance of uncertainties on results.

Public health decision making is a complicated process because of complex inputs and group decision making. Implementation of EBPH requires not only a workforce that understands and can implement EBPH efficiently but also sustained support from health department leaders, practitioners, and policy makers.

With evidence-based medicine guidelines, physicians can use proven treatment methods based on the best evidence available to develop patient-care strategies.

It is necessary to develop and promote a culture of Evidence Based Public Health approach for dealing with Emerging Diseases in the region. This will include use of Healthcare informatics, Research in Gulf Cooperation Council (GCC) countries and multidisciplinary team approach.

Multi-sectoral actions are needed to strengthen policies and improve practices that are driven by the best available evidence and knowledge. Funders often require programs to be evidence-based. Because formal public health training in the workforce is lacking, on-the-job training and skills development are needed. The need may be even greater in local health departments, where practitioners may be less aware of and slower to adopt evidence-based guidelines than state practitioners and where training resources may be more limited.(12)

There are barriers to effective implementation of evidence based public health and solutions need to be found.(13, 14)

As a first step in the EBPH process, a community assessment identifies the health and resource needs, concerns, values, and assets of a community. This assessment allows the intervention (a public health program or policy) to be designed and implemented in a way that increases the likelihood of success and maximizes the benefit to the community. Public health surveillance is a critical tool for understanding a community's health issues.

Once health needs are identified through a community assessment, the scientific literature can identify programs and policies that have been effective in addressing those needs. 
Figure 1: The practice of Evidence-Based Public Health (EBPH) is an integration of science-based interventions with community preferences for improving population health

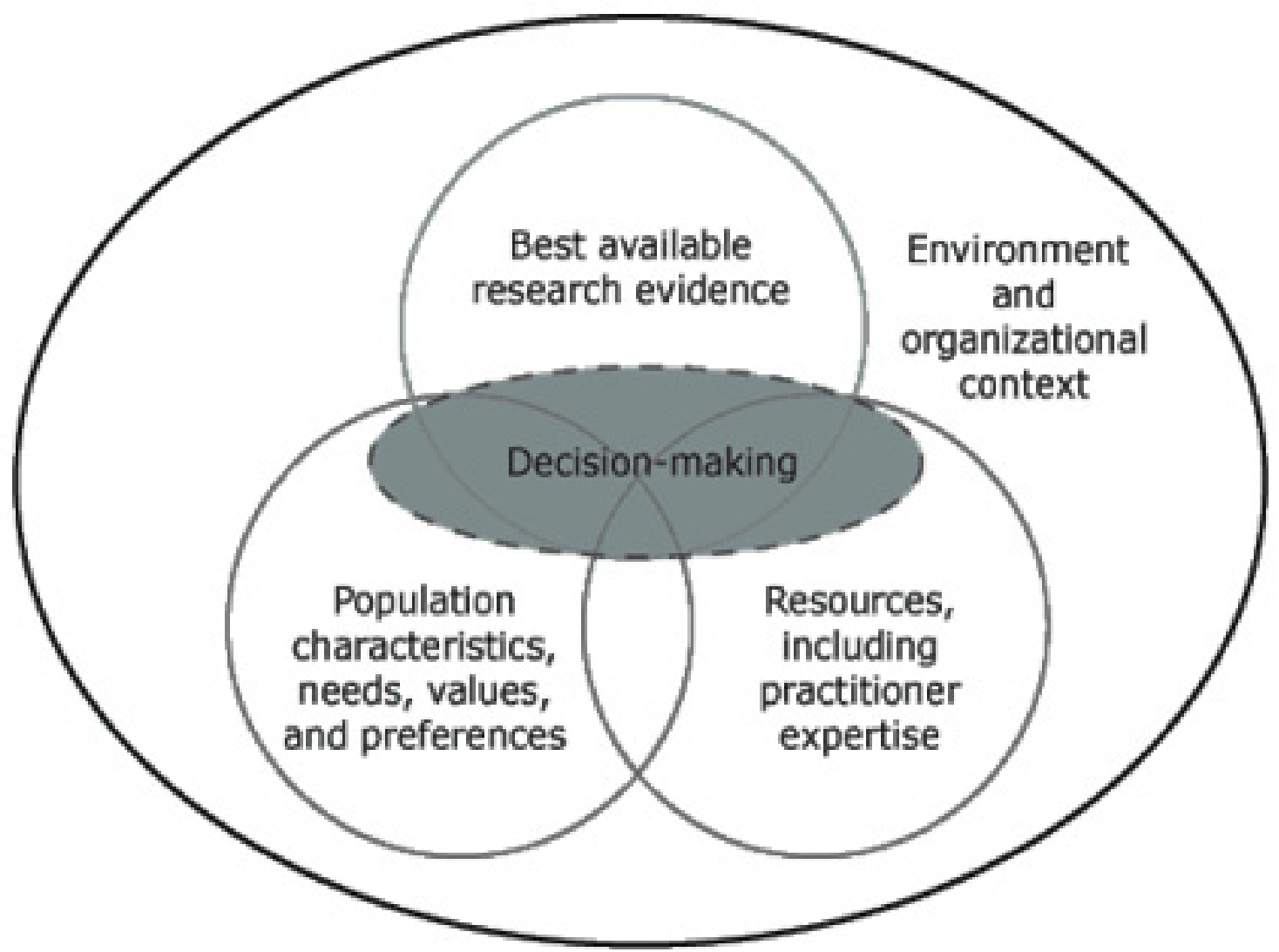

The amount of available evidence can be overwhelming; Practitioners can identify the best available evidence by using tools that synthesize, interpret, and evaluate the literature.

Systematic reviews use explicit methods to locate and critically appraise published literature in a specific field or topic area.

The products are reports and recommendations that synthesize and summarize the effectiveness of particular interventions, treatments, or services and often include information about their applicability, costs, and implementation barriers.

It is useful to consider several overarching, common characteristics of an evidence-based approach to public health practice.

- Making decisions based on the best available peerreviewed evidence (both quantitative and qualitative research);

- Using data and information systems systematically;

- Applying program planning frameworks (that often have

a foundation in behavioral science theory);

- Engaging the community in assessment and decision making;

- Conducting sound evaluation;

- Disseminating what is learned to key stakeholders and decision makers; and
- Synthesizing scientific skills, effective communication, common sense, and political acumen in making decisions.

The successful implementation of EBPH in public health practice is both a science and an art. The science is built on epidemiologic, behavioral, and policy research showing the size and scope of a public health problem and identifying interventions that are likely to be effective in addressing the problem.

The art of decision making often involves knowing what information is important to a particular stakeholder at the right time. Significant decisions in public health must balance science and art, since evidence-based decision making often involves choosing one alternative from among a set of rational choices. Interdisciplinary cooperation is necessary for success of implementation of evidence based public health initiatives. (15)

To increase the implementation of EBPH in practice settings (e.g., health departments), greater attention to administrative practices is needed, including:
1) Workforce development
2) Leadership
3) Organizational climate and culture
4) Relationships and partnerships, and
5) Financial processes 


\section{All available information must be weighed:}

- Assessment data on the magnitude of the problem, epidemiologic data on determinants

- Stakeholder opinion on the nature of the problem and acceptable solutions,

- Existing practices and traditions

- Less robust yet promising intervention evaluations, program options within budgetary constraints

- Legal considerations such as privacy laws

- Political will to address the issues

There are at least FOUR ways in which a public health program or policy may not reach stated goals for success:

- Choosing an intervention approach that's effectiveness is not established in the scientific literature;

- Selecting a potentially effective program or policy yet achieving only weak, incomplete implementation or "reach," thereby failing to attain objectives;

- Conducting an inadequate or incorrect evaluation that results in a lack of generalizable knowledge on the effectiveness of a program or policy; and

- Paying inadequate attention to adapting an intervention to the population and context of interest.

\section{Conclusion}

Numerous benefits accrue when decisions in public health are based on scientific evidence including the area of emerging diseases. By applying the concepts of EBPH, decision making and, ultimately, public health practice in emerging diseases can be improved. Public health doctors with sound clinical knowledge, skills \& competency armed with the latest research evidence are able to translate their role into effective care and public health interventions in emerging diseases.

\section{References}

1. Centers for Disease Control and Prevention. Ebola (Ebola Virus Disease). [Internet] [Cited 2015 Apr 22]. Available from: http://www.cdc.gov/vhf/ebola/index.html

2. McKibbon, K.A. et al. (1995) The medical literature as a resource for evidence based care from the Health Information Research Unit, McMaster University, Ontario, Canada.

3. Brownson, Ross C., Elizabeth A. Baker, Terry L. Leet, and Kathleen N. Gillespie, Editors. Evidence-Based Public Health. New York: Oxford University Press, 2003.

4. Kohatsu ND, Robinson JG, Torner JC. Evidence-based public health: an evolving concept. Am J Prev Med 2004; 27(5):417-21.

5. Brownson RC, Fielding JE, Maylahn CM. Evidencebased public health: a fundamental concept for public health practice. Annu Rev Public Health 2009; 30:175-201.
6. Goodman, R.M. Principles and Tools for Evaluating Community-Based Prevention and Health Promotion Programs. Journal of Public Health Management and Practice 4, no. 2 (1998): 39.

7. Brownson RC, Baker EA, Leet TL, Gillespie KN, True WR. Evidence-Based public health. 2nd edition. New York (NY): Oxford University Press; 2011.

8. Jacobs JA, Dodson EA, Baker EA, Deshpande AD, Brownson RC. Barriers to Evidence-Based decision making in public health: a national survey of chronic disease practitioners. Public Health Rep. 2010; 125(5):736-42.

9. Card AJ. Patient safety: this is public health. J Healthc Risk Manag. 2014;34(1):6-12

10. World Health Organization. WHO | Health Impact Assessment.

[Internet] [Cited 2015 Apr 22]. Available from: http://www. who.int/hia/en/

11. Mullen PD, Ramirez G. The promise and pitfalls of systematic reviews. Annu Rev Public Health 2006; 27:81102.

12. Baker EA, Brownson RC, Dreisinger M, McIntosh LD, Karamehic-Muratovic A. Examining the role of training in evidence-based public health: a qualitative study. Health Promot Pract 2009; 10(3):342-8.

13. Dodson EA, Baker EA, Brownson RC. Use of evidence-based interventions in state health departments: a qualitative assessment of barriers and solutions. J Public Health Manag Pract 2010; 16(6):E9-15.

14. Jacobs JA, Dodson EA, Baker EA, Deshpande AD, Brownson RC. Barriers to evidence-based decision making in public health: a national survey of chronic disease practitioners. Public Health Rep 2010. 125 (5):736-42.

15. Newhouse RP, Spring B. Interdisciplinary evidencebased practice: moving from silos to synergy. Nurs Outlook 2010; 58(6):309-17. 